\title{
RELATIVE IDEALS IN SEMIGROUPS, I (FADOETT'S THEOREM)
}

$\mathrm{BY}$

\author{
A. D. WALLACE (NEW ORLEANS, LA.)
}

0. In this note we show how Faucett's Theorem [2] on cut-points of the minimal ideal of a compact connected semigroup may be relativized. We also extend some results of Clifford's [1].

1. A semigroup is a non-void Hausdorff space together with a continuous associative multiplication, denoted by juxtaposition, $(x, y) \rightarrow x y$. In all that follows $S$ will denote a semigroup. A subset $T$ of $S$ will be called a subsemigroup provided $T$ is not empty and $T^{2} \subset T$. We shall generally not distinguish between $x$ and $\{x\}$ if confusion of meaning is unlikely so that we write $x A$ in place of $\{x\} A, x \cup A$ in place of $\{x\} \cup A$ and $A \backslash x$ in place of $A \backslash\{x\}$. Moreover, we omit inclusive quantifiers relative to $S$ if doing so will result in no misunderstanding.

We first define some set-valued set-functions which will depend on a subset $T$ of $S$.

In all instances $T$ will be at least a closed subsemigroup of $S$.

For $A C S$ let

$$
\begin{gathered}
L(A)=A \cup T A, \quad J(A)=A \cup T A \cup A T \cup T A T, \quad R(A)=A \cup A T, \\
H(A)=L(A) \cap R(A) .
\end{gathered}
$$

If it is desirable to call attention to $T$ we write $L(A ; T)$ for $L(A)$, and so on.

The set $A \subset S$ is termed a left $T$-ideal if $L(A) \subset A$ (i. e., if $T A \subset A$ ) and if $A$ is not empty. Similarly we define a right $T$-ideal using $R$ and a $T$-ideal using $J$. The set $A$ being non-void we see that $A$ is a $T$-ideal if and only if $T A \subset A \supset A T$.

It will be observed at once that the union and intersection (if nonvoid) of $T$-ideals of any category is again a $T$-ideal of the same category. If $A \neq \square$ (the empty set) then $L(A), J(A)$ and $R(A)$ are $T$-ideals of the appropriate category. 
In order to avoid excessive wordage we shall frequently give definitions and state propositions for only one sort of $T$-ideal and, further, if $T=S$, we shall speak of left ideal rather than left $S$-ideal, and so on.

The set $A \subset S$ is a minimal left-T-ideal if $A$ is a left $T$-ideal but if no proper subset of $A$ is a left $T$-ideal.

(1.1) If $L$ is a minimal left $T$-ideal then $L a$ is a minimal left $T$-ideal, for any $a \in S$.

Proof. Suppose that $M$ is a left $T$-ideal contained in $L a$ and let

$$
N=\{x \mid x a \in M, x \in L\}
$$

Clearly $N$ is non-void (since $M \neq \square$ ) and it is a left $T$-ideal. In fact, $T N a \subset T M \subset M$ so that, by $T L \subset L, T N \subset N$. Thus, since $L$ is minimal, $N=L$, i. e. $M \supset N a \supset L a$ and thus $L a=M$. This completes the proof.

(1.2) If there is at least one minimal left $T$-ideal then $M$, the union of all such, satisfies $T M=M \supset M S$ so that $M$ is a left $T$-ideal and a right ideal. If also there is a minimal right T-ideal and if $N$ is the union of all such then $N$ satisfies $N T=N \supset S N$ and we have

\section{$M N \subset M \cap N$}

each of these sets being a subsemigroup of $S$.

Proof. Assume that there is at least one minimal left $T$-ideal $L$. We have at once that $T M \subset M$ and by (1.1) we have $M S \subset M$. If $x \in M$ then $x \in L$ for some minimal $T$-ideal $L$ and thus $T x \subset T L \subset L$. Since $T^{2} \subset T$ we have $T(T x) \subset T^{2} x \subset T x$ so that $T x$ is a left $T$-ideal contained in the minimal left $T$-ideal $L$ and hence $T x=L$. Since $x \in L$ we have $x \in T x \subset T M$ and therefore $M \subset T M$.

As above it follows that $N T=N \supset S N$. Now the intersection and product (in this order) of a right ideal and a left ideal is a subsemigroup and the product is contained in the intersection. It is clear that an ideal of any category is a subsemigroup.

It may be that $T$ (as a semigroup in its own right) has a minimal left ideal, say $L$. Then clearly $L$ is a minimal left $T$-ideal. There may, however, be minimal left $T$-ideals which do not intersect $T$. If $S$ is the closed unit interval with its usual multiplication and if $T=\{1\}$, then any subset of $S$ is a left $T$-ideal and any element of $S$ is a minimal left $T$-ideal.

(1.3) With the notation of (1.2) suppose that $T$ has a minimal left ideal $L_{0}$ and a minimal right ideal $R_{0}$; then $M=L_{0} M=L_{0} S$ and $N=R_{0} N$ $=R_{0}$ S. Moreover, $M \cap N$ is the union of all minimal $T$-ideals and if $a \in M \cap N$, then $T a T$ is that minimal $T$-ideal which contains a and TaT $=L_{0} a R_{0}$. Finally, $M N=M \cap N$.
Proof. Let $I$ be a minimal $T$-ideal and let $a \in I$. Then $L_{0} a \subset L_{0} I$ CTI C I. Right-multiplication of $L_{0} a \subset I$ by $R_{0}$ gives $L_{0} a R_{0} \subset I$ and from this we get $L_{0} a R_{0}=I$ since $L_{0} a R_{0}$ is a $T$-ideal contained in the minimal $T$-ideal $I$. But clearly

$$
L_{0} a R_{0}=U\left\{L_{0} a r \mid r \in R_{0}\right\}
$$

is a union of minimal left $T$-ideals by (1.1) and is thus a subset of $M$. Equally, by the left-right dual of (1.1) we see that $I$ is a subset of $N$. Hence any minimal $T$-ideal is contained in $M \cap N$

Suppose now that $a \in M \cap N$; we will show that $T a T$ is a minimal $T$-ideal. If $L$ is the minimal left $T$-ideal containing $a$, then it follows readily that $L=L_{0} a$ since $L_{0} a \subset L_{0} L \subset T L \subset L$ and $T\left(L_{0} a\right) \subset\left(T L_{0}\right) \subset L_{0} a$ and $L_{0} a=L$ by the minimality of $L$. Clearly, $a \in L_{0} a$ and, in the same fasion; $a \epsilon a R_{0}$. Thus, $a \in a R_{0} \subset L_{0} a R_{0}$ and bilateral multiplication by $T$ gives $T a T \subset L_{0} a R_{0}$ since $T L_{0} \subset L_{0}$ and $R_{0} T \subset R_{0}$.

Suppose that $I$ is a $T$-ideal contained in $T a T$ and that $x \in I$, so that $x=p a q$ with $p \in L_{0}$ and $q \in R_{0}$. It follows readily that $L_{0} p=L_{0}$ and that $q R_{0}=R_{0}$ so that

$$
L_{0} a R_{0}=L_{0} p a q R_{0} \subset L_{0} I R_{0} \subset T I T \subset I .
$$

From this we conclude that $T a T$ is a minimal $T$-ideal and that TaT $=L_{0} a R_{0}$.

This is immediate: for if $x \in M \cap N$, then $x \in L_{0} x R_{0}$ as in the above argument, $L_{0} x \subset M$ and $R_{0} \subset N$ so that $x \in M N$.

(1.4) If $T$ is connected (compact) then minimal $T$-ideals of all categories are also connected (compact). If $T$ and $S$ are connected and if $T$ has a minimal left $T$-ideal and a minimal right $T$-ideal then $M, N$ and $M N=M \cap N$ are connected. If $T$ is compact then it has minimal ideals of all categories and if $S$ is compact then $M, N$ and $M N$ are olosed.

Proof. The first statement is readily disposed of since, for example, if $L$ is a minimal left $T$-ideal and if $a \in L$, then $T a=L$.

Suppose that the hypotheses in the second assertion hold - then $I=L_{0} S$ by (1.3), where $L_{0}$ is a minimal left $T$-ideal of $T$. Since $L_{0}$ is connected (by the first part) and since $S$ is connected we see that $M$ is connected. Similarly, $N$ is connected and therefore $M N$ is connected.

The preceding results relativize propositions of A.H. Clifford [1].

It is well-known (Numakura [4]) that any compact semigroup contains minimal ideals of all three categories. To prove this most quickly one takes the intersection of a maximal tower (under inclusion, by the Hausdorff Maximality. Principle) of, say, closed left ideals and it turns out that this is a minimal left ideal. The proof then proceeds as in the last argument. 
We write

$$
L_{0}(A)=\{x \mid L(x) \subset A\}
$$

and similariy for $J_{0}(A)$ and $R_{0}(A)$. Just as $L(A)$ is the smallest left $T$-ideal containing $A, L_{0}(A)$ is the largest left $T$-ideal contained in $A$, assuming of course that $L(A)$ and $L_{0}(A)$ are non-void.

As in [3] or [5] the following may be proved:

(1.5) If $A$ is closed, then $L_{0}(A)$ is closed while if $A$ is open and if $T$ is compact, then $L_{0}(A)$ is open.

(1.6) If $T$ is compact, if $A$ is compact and if there is a left $T$-ideal not containing $A$, then there is a left $T$-ideal maximal among left $T$-ideals that do not contain $A$; moreover, each such is open.

In particular, if $S$ is compact and if $S$ properly contains a left $T$-ideal then there is at least one maximal proper left $T$-ideal and each of these is open.

This result has a "dual":

(1.7) If $T$ is compact, if $A$ is closed and if some left $T$-ideal intersects $A$ then there is a minimal such and each of them is olosed. In particular, there exists a minimal left T-ideal.

2. Certain proofs will be simplified if we introduce the notion of a semigroup acting on a space.

An act is such a continuous function

$$
T \times X \rightarrow X
$$

that, employing juxtaposition to denote functional values $((t, x) \rightarrow t x)$,

(i) $T$ is a semigroup

(ii) $X$ is a non-void Hausdorff space

(iii) For any elements $t_{1}, t_{2} \in T$ and $x \in X$ we have

$$
t_{1}\left(t_{2} x\right)=\left(t_{1} t_{2}\right) x \text {. }
$$

Example I. If $S$ is a semigroup and if $T$ is a subsemigroup, then $T$ acts on $S$ by left multiplication.

Example II. Let $S$ be a semigroup and let $T$ be a subsemigroup. With the multiplication $(x, y)(z, w)=(x z, w y)$ the space $T \times T$ is a semigroup and $T \times T$ acts on $S$ in the following way:

$$
(x, y) z=x z y \text {. }
$$

Throughout this section we assume that $T$ acts on $X$ as stipulated in the definition.

It is convenient to write, for $A \subset T$ and $B \subset X$,

$$
A B=\{t x \mid t \epsilon A \text { and } x \in B\}
$$

and

$$
A^{[-1]} B=\{x \mid A x \subset B\} .
$$

A subset $I \subset X$ is subvariant if $I \neq \square$ and if $T I C I$. It is clear that $I$ is subvariant if it is not empty and if $I \subset T^{1-1} I$.

It is readily shown that if $A$ is a compact space and if $B$ is any space then the projection of $A \times B$ onto $B$ is a closed function. Employing this fact we easily prove

(2.1) If $A$ is a compact subset of $T$ and if $B$ is an open subset of $X$ then $A^{[-1]} B$ is open.

(2.2) If $T$ is connected, if $I$ is subvariant, if $B$ is a subvariant connected subset of $I$ and if $C$ is the component of $I$ containing $B$ then $C$ is subvariant.

Proof. We have

$$
T B \subset B \subset C \subset I
$$

so that

\section{$T B \subset T C \subset T I \subset I$}

and thus $T C$ is a conmected subset of $I$ which intersects $C$. Hence $O \cup T C$ is a connected subset of $I$ and thus $T C \subset C$.

We denote by $A^{*}, A^{0}$ and $F(A)$ the closure, interior and boundary of the set $A$.

(2.3) Suppose that $X$ is connected and either locally connected or compact, that $A$ is such a subset of $X$ that $A \cap T^{[-1]} A$ is a non-void proper subse of $X$ and that $C$ is a subvariant component of $A \cap T^{[-1]} A$; if $T$ is compact then the closure $C^{*}$ intersects the boundary of $A$.

Proof. If $O^{*} \cap F(A)=\square$, then $O^{*} \subset A^{0}$ and thus, since $T C \subset C$ implies $T C^{*} \subset C^{*}$, we have $T O^{*} \subset A^{0}$ and hence $C^{*} \subset T^{[-1]} A^{0}$. Thus $C^{*} \subset A^{0} \cap T^{[-1]} A^{0}$, the latter being open by (2.1). Since $A \cap T^{[-1]} A$ $\supset A^{0} \cap T^{[-1]} A^{0}$ it is clear that $C^{*}=C$ and that $O$ is a component of the latter set. But since $X$ is connected and either compact or locally connected, no component of a non-roid open proper subset of $X$ can have its closure in the subset. Thus $\sigma^{*} \cap F(A) \neq \square$.

(2.4) Let $X$ be connected and either compact or locally connected and let $T$ be compact and connected. If $z \in X$ separates two subvariant sets in $X$ then $z$ is subvariant, i. e., $T z=z$.

Proof. Suppose that $X \backslash z=U \cup \nabla$, where $U$ and $V$ are disjoint open sets and that $A \subset V$ and $B \subset V$ are subvariant. If $a \in A$ and $b \in B$, then $T a$ and $T b$ are subvariant connected sets contained in $U$ and $\nabla$. We have $T^{2} a \subset T a \subset U$ and thus $T a \subset U \cap T^{[-1]} U$, the latter being a non-void proper subvariant subset of $X$. By (2.2) the component $C$ of $U \cap T^{[-1]} U$ which contains $T a$ is subvariant and by (2.3) we know 
that $C^{*}$ intersects the boundary of $U$, i. e., $z \epsilon C^{*}$ and $T z C C^{*}$. If we argue similarly concerning $D$, the component of $V \cap T^{[-1]} V$ which contains $T b$, then $z \epsilon D^{*}$ and $T z \subset D^{*}$. It follows at once that $T z=z$.

3. In this section is the principal result of this paper, a generalization to $T$-ideals of a result due to Faucett [2]. His theorem is to the effect that if the minimal ideal of a compact connected semigroup has a cutpoint, then every element of the minimal ideal is a left zero or a right zero.

THEOREM. If $S$ is a compact connected semigroup, if $T$ is a compact conneeted subsemigroup of $S$ and if $I$ is a minimal T-ideal of $S$ then if $z$ is a outpoint of $I$ we have either $T z=z$ or $z T=z$.

Proof. Since $I C M N$ (section 1 ) it is readily seen that $I$ is the union of minimal left $T$-ideals and, as well, the union of minimal right $T$-ideals. Moreover, if $I$ contains just one minimal left $T$-ideal $L$ then $I=L$ and similarly, if $I$ contains just one minimal right $T$-ideal $R$, then $I=R$.

Suppose first that $I$ contains precisely one minimal left $T$-ideal and one minimal right $T$-ideal so that $L=I=R$ and let $L_{0}$ and $R_{0}$ be, respectively, minimal left and right ideals of $T$, or, equally, minimal left and right $T$-ideals contained in $T,(1.7)$. Let $G=R_{0} L_{0}$ so that $G$ is a group, a result of Clifford's, $[1,2.1]$. If $x \in I=L$ then $L_{0} x=L=I$, by (1.1), and thus

$$
G x=R_{0} L_{0} x=R_{0} I \subset I .
$$

On the other hand, $G x=R_{0} L_{0} x=R_{0} R$ since $L_{0} x=L=R$ and thus $G x$ is the union of minimal right $T$-ideals by the "dual" of (1.1). But $I$ is a minimal right $T$-ideal and thus $G x=R$, or, $G s=I$. We now claim that $I$ is homogeneous. For if $e$ is the unit of $G$ we have

$$
e G=G \quad \text { and } \quad e G x=G x=I
$$

so that, since $e^{2}=e$, we have $e x=x$ for each $x \in I$. It follows easily that if $g \in G$, then $x \rightarrow g x$ takes $I$ homeomorphically onto $I$ and if $x, y \in I$ then from $G x=I$ we infer that $y=g x$ for some $g \in G$. Thus $I$ is homogeneous. But then, since one point of $I$ is a cutpoint, it follows that every point of $I$ is a cutpoint contrary to a well-known result of $R$. L. Moore, see [6] or $[7$, p. 37].

Suppose then that $I$ contains more than one minimal left $T$-ideal and let $L$ be one of them which does not contain the cutpoint $z$ of $I$. We observe that $T$ acts on $I$ by left multiplication and we use the relative topology of $I$ from now on. It should be noted that for any $a \in I$ we have $T a T=I$ since $I$ is minimal and thus $I$ is a continuum.

We have $I \backslash z=U \cup V$, where $U$ and $V$ are non-void disjoint open sets and $L \subset U$. Since $T L L$ we have $L \subset U \cap T^{[-1]} U$ and this latter set is open, see section 2. By (2.2) and (2.3) the component $C$ of $U \cap T^{[-1]} U$ has the property that its closure intersects the boundary of $U$, i. e., $z \epsilon C^{*}$. But $T z \subset T C^{*} \subset C^{*} \subset U^{*}$ and $T z$ is a minimal left $T$-ideal (see (1.1)). Thus there is a minimal left $T$-ideal $L^{\prime}$ which intersects $V$ because $V \subset I \subset M \cap N=M N$. Moreover, $L^{\prime}$ cannot intersect $T z$ because $T z \subset U^{*}$ and cannot intersect $U$ because it is connected and would then have to contain $z \in T z$. Accordingly, $z$ separates $L$ and $L^{\prime}$ in $I$ and thus, by (2.4), we have $T z=z$.

It is not difficult to show that if also $z \in T$ then $T x=x$ for each $x \in I$ and thus obtain Faucett's result in toto.

I am grateful to the National Science Foundation of the United States of America for its support.

\section{REFERENCES}

[1] A. H. Clifford, Semigroups containing minimal ideals, American Journal of Mathematics 70 (1948), p. 521-526.

[2] W. M. Faucett, Topological semigroups and continua with outpoints, Proceedings of the American Mathematical Society 6 (1955), p. 748-756.

[3] R. J. Koch and A.D. Wallace, Maximal ideals in compact semigroups, Duke Mathematical Journal 21 (1954), p. 681-685.

[4] K. Numakura, On bicompact semigroups, Mathematical Journal of the Okayama University 1 (1952), p. 99-108.

[5] A. D. Wallace, Struct Ideals, Proceedings of the American Mathematical Society 6 (1955), p. 634-638.

[6] - A note on mobs, Anais da Academia Brasileira de Ciências 24 (1952), p. $329-334$.

[7] R.L. Wilder, Topology of manifolds, New York 1949.

THE TULANE UNTVERSITY OF LOUISIANA

Reģu par la Rédaction le 22. 3. 1961 